\title{
Coordenação do cuidado no Consultório na Rua no município do Rio de Janeiro: romper barreiras e construir redes
}

\author{
Coordination of care in the 'Street Clinic' in Rio de Janeiro: breaking \\ barriers and building networks
}

Amanda Rodrigues dos Santos ${ }^{\mathbf{1}}$, Patty Fidelis de Almeida ${ }^{\mathbf{1}}$

DOI: $10.1590 / 0103-1104202112906$

RESUMO O objetivo do artigo foi caracterizar arranjos e ações de coordenação do cuidado desenvolvidos pelo Consultório na Rua (CnaR). Foi realizado estudo qualitativo a partir de observação participante e entrevistas com profissionais do CnaR e serviços de referência em um município de grande porte. A coordenação do cuidado foi analisada nas dimensões horizontal e vertical. Os resultados mostram que a coordenação horizontal é fortalecida pelo acolhimento, pela presença e insistência da equipe em estar nas ruas, em reunir-se formal e informalmente, pela plasticidade da agenda e pelos diversos agenciamentos para garantia da estada dos usuários em outros espaços dentro e fora do setor saúde. O desenvolvimento de ações intersetoriais enfrenta diferentes concepções das políticas e do direito à saúde entre os órgãos governamentais. A coordenação entre níveis é ainda mais frágil pela fragmentação dos serviços, ausência de comunicação profissional, exigência de documentos, desresponsabilização dos demais serviços e precariedade dos vínculos laborais que impediam a tessitura de relações duradouras na rede. Iniciativas de coordenação do cuidado pelo CnaR reafirmam seu papel estratégico na composição de redes, negociações, tensões e desconfortos que pode provocar ao reconduzir, ainda que de forma parcial, excluídos ao campo da cidadania e do direito à saúde.

PALAVRAS-CHAVE Atenção Primária à Saúde. Pessoas em situação de rua. Prestação integrada de cuidados de saúde.

1 Universidade Federal Fluminense (UFF), Instituto de Saúde Coletiva (ISC) Niterói (RJ), Brasil. pattyfidelis@id.uff.br

\begin{abstract}
The aim of this paper is to characterize the arrangements and actions of coordination of care developed by the 'Street Clinic' (Consultório na Rua/CnaR). A qualitative study was carried out based on participant observation and interviews with $C n a R$ professionals and reference services in a large city. The care coordination was analyzed in the horizontal and vertical dimensions. The results show that the horizontal coordination is strengthened by the reception, presence, and insistence of the team in being on the streets, in meeting formally and informally, by the schedule flexibility, and by the various actions to ensure the users' permanence in other spaces inside and outside the healthcare sector. The development of intersectoral actions faces different conceptions of the policies and the right to health among governmental agencies. Coordination between levels is even more fragile due to the fragmentation of services, lack of professional communication, document requirements, non-accountability of other services, and precarious labor relations that prevent the construction of long-lasting relationships. CnaR's care coordination initiatives reaffirm its strategic role in the weaving of networks, negotiations, tensions and discomforts that it can provoke by bringing, even partially, excluded people back to the field of citizenship and the right to health.
\end{abstract}

KEYWORDS Primary Health Care. Homeless persons. Delivery of health care, integrated. 


\section{Introdução}

A Estratégia Saúde da Família (ESF) representa o principal modelo de Atenção Primária à Saúde (APS) no País e, desde os anos 2000, é responsável pela ampliação do acesso à saúde a milhões de brasileiros ${ }^{1}$. Reconhecendo as especificidades e as desigualdades de territórios e populações, a Política Nacional de Atenção Básica (PNAB), em sua primeira revisão, no ano de $2011^{2}$, incorporou um conjunto de configurações singulares de equipes de Atenção Básica (AB), com financiamento específico, reconhecendo que a garantia da atenção à saúde de todo cidadão é responsabilidade do Sistema Único de Saúde (SUS), sobretudo da APS.

A inclusão das pessoas em situação de rua na PNAB $2011^{2}$ reflete uma tentativa de enfrentamento das contradições e complexidades do estar na rua em suas dimensões política, econômica e social. Destaca-se que experiências de cuidados a essas populações antecedem sua incorporação à PNAB, sendo parte, até então, da Política Nacional de Saúde Mental. A inserção na agenda governamental foi precedida e reivindicada por um conjunto de movimentos sociais e experiências prévias, entre as quais o projeto-piloto Consultório de Rua, em 1999, em Salvador, Bahia, criado para atender crianças e adolescentes que se encontravam na rua e sob uso de álcool e outras drogas ${ }^{3}$. Em 2004, o Consultório de Rua foi implementado, na mesma cidade, no primeiro Centro de Atenção Psicossocial para atendimento de álcool e outras drogas (Caps-AD). Outro marco importante para a incorporação das pessoas em situação de rua nas políticas sociais foi a 'Pesquisa Nacional da População de Rua', realizada em $2008^{4}$.

A mudança para Consultório 'na' Rua na PNAB 2011 não representa apenas uma mudança de nomenclatura, mas nas diretrizes estratégicas desse dispositivo, atendendo, ainda, à prioridade das políticas nacionais, de prevenção do consumo e da dependência de crack, álcool e outras drogas ${ }^{3}$. A política nacional define a modalidade de equipe 'Consultório na Rua' (CnaR), em estreita relação com os dispositivos da rede de saúde mental que compõe a Rede de Atenção Psicossocial (Raps). O CnaR é, nesse sentido, uma equipe de $\mathrm{AB}$ exclusivamente dirigida às pessoas em situação de rua, para "ampliar o acesso desses usuários à rede de atenção e ofertar de maneira mais oportuna atenção integral à saúde"2(62).

Estudos revelam que a maior parte das pessoas em situação de rua procuram os serviços de emergência para condições que poderiam ser resolvidas na APS ${ }^{5,6}$. Além disso, garantir o acesso aos serviços de saúde tem sido um desafio em várias partes do mundo. Borysow et al.7, em análise comparada, destacaram que, apesar das diferenças estruturais nas políticas de saúde em países como Brasil, Portugal e Estados Unidos da América (EUA), a principal barreira de acesso da população em situação de rua é a inadequação dos serviços às especificidades e necessidades desse grupo.

É inegável o avanço representado pela inclusão das pessoas em situação de rua na PNAB, muitas vezes consideradas 'descartáveis urbanos' em uma perspectiva higienista, que contribui para mitigar o acesso aos serviços de saúde ${ }^{\mathbf{8}}$. Ainda assim, muitos são os desafios. No Brasil, as diretrizes assumidas pelas consecutivas $\mathrm{PNAB}^{2,9}$ ancoram-se, de forma geral, em noções convencionais de território, família e domicílio que servem de base ao trabalho desenvolvido pelas equipes de $\mathrm{AB}$. Ao abalar alicerces tradicionais que orientam o trabalho em saúde, o cuidado às pessoas em situação de rua desafia profissionais e gestores a (re)criarem processos e desenhos de Redes de Atenção à Saúde (RAS) capazes de garantir a esses usuários os mesmos atributos que caracterizam uma APS abrangente.

Um conjunto de estudos avaliam alcances e desafios, principalmente dos atributos essenciais da APS no País ${ }^{10,11}$. Em relação ao CnaR, as investigações centram-se, sobretudo, na implementação do dispositivo e inovações das práticas de saúde, acolhimento, escuta qualificada e atenção às vulnerabilidades ${ }^{3,4,12-18}$. Poucos trabalhos abordam o atributo da coordenação do cuidado no trabalho desenvolvido pelas equipes de $\mathrm{CnaR}^{15,17}$, sendo este um tema pendente. Além disso, em países de 
alta renda da União Europeia e EUA, a população em situação de rua apresenta altas taxas de mortalidade prematura, sobretudo por suicídio e lesões não intencionais, além de prevalência aumentada para uma variedade de doenças infecciosas (tuberculose, HIV/Aids, hepatites), transtornos mentais e uso indevido de substâncias psicoativas, se comparada à população geral ${ }^{5}$. Constatam-se, nesses países, envelhecimento da população em situação de rua e aumento das taxas de doenças não transmissíveis, mesmo em idade mais precoce, exigindo dos serviços de saúde integração entre as especialidades médicas ${ }^{5}$. Nesse sentido, o complexo perfil de multimorbidade confere ainda mais importância às ações de coordenação que viabilizem o trânsito e o cuidado ao longo da RAS.

Interessou, neste estudo, caracterizar quais são os arranjos e ações de coordenação do cuidado desenvolvidos por profissionais de saúde do CnaR para efetivar o cuidado integral e continuado a partir da noção de saúde como direito. O conceito de coordenação adotado considera duas dimensões, embora se reconheçam diversas interfaces ${ }^{19,20}$. A primeira é a coordenação entre níveis assistenciais ou coordenação vertical que trata da integração de serviços de diferentes densidades tecnológicas ${ }^{21}$; a segunda é à coordenação horizontal que compreende arranjos e iniciativas que fomentem a integração entre profissionais, serviços e ações de saúde em um mesmo nível de atenção e no território, incluindo as ações intersetoriais ${ }^{22}$. Em uma concepção ampliada do conceito de coordenação horizontal, serão considerados também elementos relativos à posição da ESF na RAS, sobretudo, na configuração da porta de entrada, considerando-se que sem a entrada no sistema de saúde não se colocaria a possibilidade de coordenação ${ }^{21,22}$.

\section{Material e métodos}

Trata-se de um estudo qualitativo, de caráter exploratório, que utilizou, como instrumento de pesquisa, a observação participante para acompanhamento do cotidiano do trabalho e a realização de entrevistas semiestruturadas com profissionais de uma equipe de CnaR, entre os meses de agosto e novembro de 2019. Também foram realizadas entrevistas com trabalhadores de um serviço de APS que ofertava cuidado às pessoas em situação de rua no mesmo território. O perfil dos entrevistados e códigos de identificação encontram-se no quadro 1.

\begin{tabular}{|c|c|c|}
\hline Código & Formação/Função & Tempo na função \\
\hline P1 & $\begin{array}{l}\text { Serviço Social - CnaR } \\
\text { Pós-graduação em Saúde da Família e Serviço social }\end{array}$ & 4 anos \\
\hline P2 & $\begin{array}{l}\text { Enfermagem - CnaR } \\
\text { Pós-graduação em Pediatria Neonatal }\end{array}$ & 9 meses \\
\hline P3 & $\begin{array}{l}\text { Técnico de Processamento de dados - CnaR } \\
\text { Agente Social de Saúde }\end{array}$ & 5 anos \\
\hline P4 & $\begin{array}{l}\text { Psicologia - CnaR } \\
\text { Pós-graduação em Atenção Psicossocial e em Álcool e Outras Drogas }\end{array}$ & 3 anos e 11 meses \\
\hline P5 & $\begin{array}{l}\text { Medicina - CnaR } \\
\text { Pós-graduação em Gestão, Saúde da Família e Atenção Domiciliar }\end{array}$ & 1 ano e 3 meses \\
\hline P6 & $\begin{array}{l}\text { Medicina - CnaR } \\
\text { Pós-graduação em Medicina da Família }\end{array}$ & 6 meses \\
\hline P7 & $\begin{array}{l}\text { Serviço Social - CnaR } \\
\text { Pós-graduação em Políticas Sociais }\end{array}$ & 1 ano e 6 meses \\
\hline
\end{tabular}


Quadro 1. (cont.)

\begin{tabular}{lll}
\hline Código & Formação/Função & Tempo na função \\
\hline P8 & Agente Social de Saúde Magistério - CnaR & 5 anos \\
& Agente Social de Saúde & 3 anos \\
P9 & Enfermagem - CnaR & \\
& Pós-graduação em Saúde da Família & \\
& Gerente da Unidade & $+/-1$ ano \\
P10 & Medicina - Centro de Saúde Escola & \\
& Pós-graduação em Saúde da Família & \\
P11 & Médico e Gerente da Unidade & 6 meses \\
\hline
\end{tabular}

Fonte: Elaboração própria.

O estudo foi realizado no município do Rio de Janeiro, que contava com sete equipes de $\mathrm{CnaR}^{23}$. Foi eleita de forma intencional uma equipe do centro da cidade, região em que ocorreram os primeiros ensaios de práticas de cuidado em saúde às pessoas em situação de rua, derivada da experiência do Projeto Meio-Fio, desenvolvido pelos Médicos Sem Fronteiras, entre 2000 e $2004^{16}$. Além disso, na área central da cidade, encontra-se um dos maiores contingentes de pessoas em situação de rua no município.

Sob inspiração da etnografia, realizou-se observação participante, técnica que permitiu estabelecer uma relação mais próxima, interativa, frequente e prolongada com os sujeitos da pesquisa durante certo período, ancorada na possibilidade de um contato com a produção de práticas situadas ${ }^{\mathbf{2 4}}$ no cotidiano das ruas. Nessa modalidade de investigação, o pesquisador é o principal recurso, e os dados são recolhidos por meio das observações e conversas informais registadas no diário de campo, além de entrevistas com profissionais de saúde ${ }^{25}$. A observação foi realizada a partir do acompanhamento de todas as atividades realizadas pela equipe do CnaR no Centro Municipal de Saúde (CMS) no qual está alocada e nos espaços fora do CMS, em turnos alternados, entre agosto e outubro de 2019, somando-se cerca de 70 horas. Foi possível observar o conjunto das ações desenvolvidas, o que permitiu incorporar novas dimensões à análise da coordenação. Ademais, as vivências registradas no diário de campo foram, posteriormente, categorizadas.

Outra fonte de dados foram 11 entrevistas semiestruturadas, com todos os profissionais do CnaR (8), incluindo a gerência do serviço (1) e profissionais do Centro de Saúde Escola (2), localizado no mesmo território do CnaR. As entrevistas realizadas nos respectivos locais de trabalho, com duração aproximada de 40 minutos, foram gravadas em aparelho de áudio e transcritas na íntegra.

Para a produção dos resultados, foi realizada análise temática de todo material seguindo as etapas de categorização, descrição e interpretação. A fase de pré-análise envolveu a leitura flutuante de todas as entrevistas e diário de campo, buscando-se identificar as dimensões envolvidas no trabalho do CnaR. Em um segundo momento, foi realizada leitura exaustiva das entrevistas e do diário de campo para descrição da coordenação vertical e horizontal do cuidado. A observação e as entrevistas foram interpretadas à luz de uma matriz de análise construída a partir de estudos nacionais sobre coordenação do cuidado $\mathbf{2 1}^{222,26}$, revisitada e adaptada a partir dos estudos sobre o dispositivo CnaR $\mathrm{R}^{\mathbf{4}, 18,27-29}$, composta por variáveis que se interrelacionam. Incorporaram-se, à matriz final, categorias emergentes a partir da experiência dos sujeitos envolvidos (quadro 2). 
Quadro 2. Dimensões e categorias da coordenação do cuidado no Consultório na Rua, 2019

\begin{tabular}{|c|c|c|}
\hline $\begin{array}{l}\text { Modalidade } \\
\text { coordenação }\end{array}$ & Dimensões & Variáveis \\
\hline \multirow{16}{*}{$\begin{array}{l}\text { Coordenação } \\
\text { horizontal }\end{array}$} & \multirow[t]{7}{*}{ Porta de entrada } & Acesso ao CnaR \\
\hline & & Acolhimento \\
\hline & & Vínculo \\
\hline & & Cadastramento \\
\hline & & Busca ativa \\
\hline & & Atividades itinerantes com ações na rua \\
\hline & & Organização da agenda de trabalho \\
\hline & \multirow{4}{*}{$\begin{array}{l}\text { Integração entre os } \\
\text { serviços de saúde } \\
\text { no território }\end{array}$} & Estratégias de articulação da RAS e Raps no território \\
\hline & & Compartilhamento de casos com outros serviços territoriais \\
\hline & & Supervisão e apoio institucional \\
\hline & & Reunião de equipe CnaR \\
\hline & \multirow[t]{5}{*}{ Intersetorialidade } & Ações intersetoriais e parcerias desenvolvidas pela equipe CnaR \\
\hline & & $\begin{array}{l}\text { Estratégias de integração entre Seguridade Social, rede de saúde e rede de } \\
\text { assistência social, Organizações Não Governamentais etc. }\end{array}$ \\
\hline & & Construção de Projeto Terapêutico Singular com participação intersetorial \\
\hline & & Reuniões ampliadas de equipe \\
\hline & & Fóruns Intersetoriais \\
\hline \multirow[t]{7}{*}{$\begin{array}{l}\text { Coordenação } \\
\text { vertical }\end{array}$} & \multirow{4}{*}{$\begin{array}{l}\text { Oferta e acesso à } \\
\text { atenção especiali- } \\
\text { zada e hospitalar }\end{array}$} & $\begin{array}{l}\text { Oferta e acesso de serviços na RAS disponíveis para a população em situação } \\
\text { de rua }\end{array}$ \\
\hline & & Definição de fluxos assistenciais na RAS \\
\hline & & $\begin{array}{l}\text { Estratégias para transporte sanitário da população em situação de rua aos } \\
\text { demais serviços da RAS }\end{array}$ \\
\hline & & $\begin{array}{l}\text { Monitoramento das referências realizadas pelos profissionais do CnaR (Sisreg, } \\
\text { especialidades e hospitais) }\end{array}$ \\
\hline & \multirow{3}{*}{$\begin{array}{l}\text { Instrumentos de } \\
\text { integração entre } \\
\text { níveis e continuida- } \\
\text { de do cuidado }\end{array}$} & $\begin{array}{l}\text { Iniciativas para sensibilização dos demais profissionais da RAS em relação ao } \\
\text { acesso à saúde da população vivendo em situação de rua }\end{array}$ \\
\hline & & Estratégias de comunicação interprofissional \\
\hline & & $\begin{array}{l}\text { Acompanhamento de usuários em consultas de especialidades clínicas, exa- } \\
\text { mes, rede de urgência e emergência e internação hospitalar }\end{array}$ \\
\hline
\end{tabular}

RAS - Rede de Atenção à Saúde; Sisreg - Sistema de Regulação. 
O estudo foi aprovado pelos Comitês de Ética em Pesquisa da Universidade Federal Fluminense (Parecer 3.377.781) e do município do Rio de Janeiro (Parecer 3.496.162) e aprovado no Centro de Estudos da Área Programática 1.0.

\section{Resultados}

\section{Coordenação horizontal do cuidado no Consultório na Rua: desvelando as ações no território}

\section{PORTA DE ENTRADA}

A equipe de CnaR estava instalada no quarto andar de um prédio de dez andares de um CMS, conforme destacado anteriormente, com funcionamento de segunda a sexta-feira, das 8 horas às 17 horas. Nesse espaço, ela utilizava três salas distintas: uma sala chamada de 'acolhimento', na qual eram realizados o cadastro e os atendimentos feitos pelas agentes sociais de saúde, atividades estas que também eram desenvolvidas na rua; uma sala da equipe 'técnica', dividida entre os profissionais; uma sala de reunião que também funcionava como um espaço comum a todos os profissionais para café, almoços. $\mathrm{O}$ acesso a essa estrutura acontecia por meio de escadas e elevador.

Apesar de ser uma unidade próxima aos territórios de maior concentração de pessoas em situação de rua, apenas a proximidade geográfica não era suficiente para garantia do acesso. $\mathrm{O}$ fato de o CnaR estar sediado em um CMS gerava tensões entre os demais usuários e profissionais da unidade, que se queixavam da presença de pessoas em situação de rua, mal-estar que se configurava como uma primeira barreira de acesso. Para amenizar os conflitos, a direção do CMS estabeleceu que os atendimentos do CnaR deveriam acontecer apenas na rua, o que se tornou perceptível no esvaziamento do quarto andar. Em uma das situações observadas, um usuário sob efeito do uso de álcool, que procurou a equipe do CnaR, falava muito alto e foi retirado de forma violenta pelo segurança do CMS, após ter o atendimento negado. Tal situação, claramente, representou mitigação do acesso.

Na sala do 'acolhimento', acontecia a maior parte dos atendimentos do CnaR, sendo considerada uma importante porta de entrada. Durante o acolhimento, ocorriam cadastro, entrega de medicamentos, esclarecimento de dúvidas, organização da fila de curativos e outras atividades realizadas pelos agentes sociais. Na sala ocupada pela equipe 'técnica' (composta pelos médicos, enfermeiro, psicólogo e assistente social), também eram realizados atendimentos, especialmente médicos, embora com pouca frequência.

Durante o acolhimento, na rua e no CMS, percebeu-se preocupação da equipe em esclarecer as ofertas de cuidado e colocar-se à disposição dos usuários. A abordagem ancorava-se na redução de danos, com enfoque na 'corresponsabilidade' do usuário com seu cuidado, respeitando sua liberdade e suas escolhas. Esse momento também era marcado por tensões e desconfiança por parte dos usuários que, muitas vezes, recusavam-se a serem registrados por problemas com a justiça. É importante ressaltar que a única forma de identificação da equipe CnaR era o crachá, para que não fossem confundidos com profissionais de outros dispositivos públicos.

As ações na rua e busca ativa foram dispositivos essenciais para garantia de acesso ao cuidado, configurando-se em outra estratégia de porta de entrada, em uma perspectiva de deslocamento das práticas do CMS para uma postura ativa e de articulação com o território. A maior parte das ações realizadas pelo CnaR ocorriam na rua, como: entrega de referência (Sistema de Regulação - Sisreg), marcação de consulta, curativos, escuta qualificada, atendimentos em equipe e atendimentos individuais, pactuações com usuários, marcação de exames como preventivo e testes rápidos. O planejamento era flexível e variava de acordo com as 
necessidades dos usuários, das condições de clima/tempo e da disponibilidade do carro/van.

$O$ vínculo se constituía, sobretudo, pela insistência da equipe em encontrar-se com os usuários no território, considerando o perfil nômade das pessoas que vivem em situação de rua. Para tal, o planejamento das ações e da agenda de forma flexível e sensível às necessidades dos usuários era uma característica importante que contribuía para um modo de operar as práticas de cuidado em saúde que considerava a dinamicidade das demandas do 'estar na rua' com vistas à garantia do acesso.

\section{INTEGRAC̣ÃO ENTRE OS SERVIC̣OS DE SAÚDE DO TERRITÓRIO}

O CnaR compõe a Raps, entretanto, não havia Caps no mesmo território. Os serviços de referência em saúde mental eram o Centro Psiquiátrico do Rio de Janeiro (CPRJ); a Unidade de Problemas Relacionados a Álcool e outras Drogas e o Núcleo de Estudos e Pesquisas em Atenção ao Uso de Drogas. A equipe também contava com Programa de Estudos e Assistência ao Uso Indevido de Drogas, localizado em outro território, o que dificultava o acesso dos usuários. Entre esses serviços, apenas o CPRJ compunha a Raps, os demais faziam parte de programas universitários.

Apesar da existência de referência, a articulação do CPRJ com o CnaR era bastante frágil. Tais serviços apresentavam dificuldades em acolher as demandas mais complexas das pessoas em situação de rua. O CPRJ, referência para agravos relacionados ao uso de álcool e outras drogas, funcionava de forma apartada de rede SUS, refratário a tentativas do CnaR em estabelecer mecanismos de referência e contrarreferência ou mesmo de comunicação informal.

Além dos serviços de saúde mental, a integração com as outras unidades de APS do território também se expressou de forma fragilizada. Havia pouca comunicação entre CnaR e as demais equipes da ESF no território. Durante o trabalho de campo, foi possível acompanhar a aproximação de uma unidade de APS escola, pertencente a uma universidade privada. A partir daí, foram propostas iniciativas para o compartilhamento de casos entre as equipes.

Em relação ao apoio institucional ou alguma modalidade de supervisão ofertada pela coordenação central do CnaR, apesar de haver um apoiador institucional de referência, não havia um trabalho contínuo ou aproximação. Em uma reunião de equipe, observou-se que o apoiador se ocupava da função de dar informes, provocando nos profissionais a percepção de não serem, de fato, apoiados pelo nível central.

A reunião de equipe foi um momento importante de organização da agenda e de pactuação de estratégias e ações para garantir o acesso dos usuários aos outros serviços, em especial hospitalares. Era realizada no CMS, semanalmente, e representava o único momento com a participação de todos os profissionais, visto que somente os agentes sociais e o enfermeiro possuíam carga de 40 horas semanais. O quadro mensal de organização do trabalho era rediscutido na reunião de equipe e alterado de acordo com as demandas emergentes. Durante a reunião, havia discussão de pelo menos um caso, pois era um momento em que todos os profissionais estavam presentes. Ainda que esse fosse o espaço formal de encontro da equipe, no início e no final de cada dia ou durante as refeições, ocorriam reuniões informais, que também funcionavam como momentos de compartilhamento.

\section{INTERSETORIALIDADE}

A equipe do CnaR conhecia a maior parte dos serviços que atendiam pessoas em situação de rua, no entanto, não havia um fluxo formalizado que estabelecesse as relações entre os equipamentos. Durante os atendimentos, informavam aos usuários sobre serviços no território, como banho gratuito, alimentação oferecida por instituições religiosas e como acessar o Núcleo de Defesa dos Direitos para tratar de questões documentais. As ações 
intersetoriais eram desenvolvidas, sobretudo, por profissionais mais específicos, como a assistente social e a psicóloga para casos considerados de 'demanda social', como retirada de documentação e benefícios sociais.

Apesar do número expressivo de instituições que atendiam pessoas em situação de rua, incluindo serviços da assistência social, Organizações Não Governamentais, igrejas etc., as ações intersetoriais do CnaR eram incipientes. A fragilidade dos vínculos laborais promovia desmotivação e alta rotatividade de profissionais em todos os serviços, promovendo descontinuidade das ações.

Outra questão relevante foram as concepções conflitantes de políticas públicas entre órgãos governamentais. Em 5 de setembro de 2019, o governo municipal lançou o programa Resgate Solidário, que incluiu diferentes setores como Secretaria Municipal de Saúde, Coordenadoria Municipal de Políticas sobre Drogas, Guarda Municipal, Comlurb e Secretaria de Ordem Pública. O objetivo do programa era realizar ações noturnas de recolhimento das pessoas em situação de rua e/ou usuários de álcool e outras drogas e levá-las para instituições como as Comunidades Terapêuticas, o que entrava em conflito com a concepção de cuidado e direito à saúde do CnaR. As internações compulsórias, que envolviam agentes da assistência, da Guarda Municipal e a empresa carioca de coleta de lixo para retirada e descarte dos pertences, eram compreendidas pela equipe como práticas higienistas e violentas.

Além do Resgate Solidário, outras ações da Secretaria de Segurança que também abordavam pessoas em situação de rua interferiam diretamente no trabalho do CnaR. Em alguns momentos, a equipe evitava atendimentos quando havia a presença da Guarda Municipal ou da polícia, para que não fosse confundida e identificada a tais ações, com vistas a preservar o vínculo com os usuários. Percebeu-se que os locais com alta concentração de pessoas em situação de rua esvaziavam-se após tais abordagens.
No município, está instituído o 'Fórum municipal de pessoas em situação de rua', com frequência mensal, que tem como objetivo discutir políticas públicas para essa população. Era composto por pesquisadores, movimento social das pessoas em situação de rua, CnaR, representantes da Secretaria de Assistência Social, Secretaria de Saúde, além de convidados como pesquisadores e estudantes. O Fórum apresentou-se como um espaço potente para troca de experiências, mas também revelou o desconhecimento de outros serviços em relação ao trabalho do CnaR, que era considerado, com frequência, o único serviço responsável pela saúde das pessoas em situação de rua, como se fosse um serviço especializado, e não parte da AB. Nesse sentido, o papel do CnaR na ampliação do acesso à atenção integral à saúde das pessoas em situação de rua, assim como das que fazem uso de álcool e outras drogas, a partir da articulação com a RAS, a Raps e as redes intersetoriais, precisava ser cotidianamente ratificado e retificado.

\section{Coordenação vertical do cuidado: o desafio da integração entre níveis assistenciais no CnaR}

\section{OFERTA E ACESSO À ATENÇÃO ESPECIALIZADA}

O acesso aos serviços de atenção especializada era regulado pelo Sisreg, mesmo sistema utilizado para pessoas domiciliadas, apresentando as mesmas dificuldades, como os longos tempos de espera para agendamento e realização de consultas e exames especializados. A equipe do CnaR monitorava a realização das referências; e quando se aproximava a data do procedimento, eram realizadas buscas para avisar ou lembrar aos usuários os dias e horários do agendamento. Como os tempos de espera eram longos, muitas vezes, a equipe não conseguia encontrá-los em função da grande circulação pela cidade.

A maternidade foi apontada como o serviço de maior articulação e com maior comunicação 
entre os profissionais, que compartilhavam informações sobre pré-natal e altas após o parto.

A integração com os serviços que compõem a rede de urgência e emergência era pontual e acontecia em casos de demandas específicas. Havia dificuldade de esses serviços acolherem as necessidades das pessoas em situação de rua, e, por fim, acabavam por reencaminhar ou orientar os usuários a procurarem cuidados na equipe do CnaR. Além da Unidade de Pronto Atendimento e dos hospitais, a rede de urgência e emergência contava com os Centros de Emergência Regional (CER), unidades instaladas ao lado de grandes hospitais com a função de atender às demandas de menor complexidade. Também realizava triagem dos casos, referenciando-os para a APS, rede especializada ou, quando necessário, para internação. Durante a observação, foram acompanhados alguns casos de atendimentos a usuários que chegavam ao CnaR por indicação do CER, mesmo se tratando de situações graves de saúde.

Os usuários não costumavam procurar os serviços hospitalares devido às dificuldades de acesso e falta de documentação, o que impossibilitava o exercício do direito ao acesso universal à saúde no SUS e amplificava as inúmeras iniquidades às quais estava submetida essa população. Nesses casos, a equipe do CnaR realizava a regulação via 'vaga zero' para garantia do acesso imediato. Alguns profissionais ressaltaram que a interdição do acesso aos serviços de saúde pela exigência de documentos era mais uma violência perpetrada pelo estado que, contraditoriamente, também empreendia ações que acabavam por incorrer na perda de documentos.

A oferta de retaguarda terapêutica obedecia aos mesmos fluxos da população geral, sem adequações em função das particularidades e vulnerabilidades desse grupo. Muitos serviços de referência localizavam-se em áreas distantes do território no qual as pessoas em situação de rua viviam, constituindo importante barreira de acesso geográfica e financeira.

As estratégias utilizadas pela equipe do CnaR para garantia à referência e de alguma coordenação eram traçadas caso a caso, a depender do grau de autonomia de cada usuário.
Por exemplo, uma das ações consistia em acompanhá-los à consulta, utilizando-se o veículo do CnaR, ou oferecer o cartão RioCard que garantia gratuidade no transporte municipal para pessoas com deficiência e doenças crônicas.

Não havia compartilhamento do cuidado com outros serviços e profissionais da RAS. $\mathrm{O}$ acesso aos demais níveis assistenciais era marcado por tensões em que a certeza da realização da consulta só era possível na presença de pelo menos um profissional do CnaR, deslocado ao lugar de único responsável pela atenção à saúde das pessoas em situação de rua na rede, em constante reafirmação, com os demais profissionais e serviços, que se tratava de direito à saúde.

\section{INSTRUMENTOS DE INTEGRAÇÃO ENTRE NÍVEIS E CONTINUIDADE DO CUIDADO}

Não havia estratégias estabelecidas para a sensibilização dos demais profissionais da RAS em relação ao acesso das pessoas em situação de rua. Embora inciativas tenham sido desenvolvidas, deixaram de ocorrer em função da sobrecarga assistencial da equipe CnaR e da alta rotatividade de profissionais em toda a RAS. Contudo, durante o acompanhamento cotidiano das atividades, foi possível observar que a sensibilização ocorria 'em ato', nos encontros com outros profissionais da rede. O principal desafio residia no convencimento de que o acesso aos serviços do SUS configurava-se como um direito de todo cidadão, e não como caridade.

As estratégias de comunicação interprofissional variavam de serviço para serviço, mas, segundo os profissionais do CnaR, dificilmente aconteciam formalmente. Os principais instrumentos eram operacionalizados por vias informais, principalmente pelo WhatsApp. Contatar profissionais conhecidos para facilitar o acesso a outros serviços de referência também ocorria.

O vínculo da equipe CnaR com os usuários era o principal instrumento de coordenação do cuidado e garantia da integralidade possível. 
Por meio do vínculo, estabelecia-se a continuidade do cuidado e o acompanhamento nas consultas especializadas ou internação. Além disso, na persistência da equipe na busca pelos usuários no território, viabilizavam-se alguma resolutividade da atenção por meio da entrega de medicamentos e resultados de exames.

\section{Discussão}

O CnaR como dispositivo da APS representa, no contexto do estudo, a principal porta de entrada do SUS para a população em situação de rua. Contudo, sua maior inserção na RAS possibilitaria alguma coordenação e prestação de cuidados mais integrais, ainda que em outros termos. A aposta seria também de enfrentamento de preconceitos e estigmas presentes nos serviços e prática dos profissionais de saúde ${ }^{3}$. Os resultados do estudo mostram que a dimensão da coordenação horizontal, mais dependente da atuação da equipe, é fortalecida pelo acolhimento dentro e fora do serviço, pela presença e insistência da equipe em estar nas ruas, em reunir-se formal e informalmente, pela plasticidade da agenda e pelos diversos agenciamentos para garantia da estada dos usuários em outros espaços dentro e fora do setor saúde. Tais dispositivos foram potentes para abrir a porta de entrada do SUS, que sofreu várias interdições, inclusive pela limitação da circulação de seus usuários pela unidade que sedia o CnaR. A intersetorialidade também não acontece pelas vias mais comuns da ESF e enfrenta, além das dificuldades do trabalho em parceria, diferentes concepções de cuidado, de direito à saúde e formas de operar as políticas entre os agentes públicos, com violação sistemática dos direitos das populações mais vulneráveis, em atos e políticas públicas. A coordenação entre níveis assistenciais, mais dependente das relações entre os serviços da RAS, é ainda mais frágil pela própria fragmentação dos serviços e ausência de comunicação profissional, problemas agravados pela exigência de documentos e desresponsabilização dos demais serviços pelo cuidado à população em situação de rua. A precariedade dos vínculos e condições laborais também fragiliza a tessitura de relações duradouras que permitiriam alguma coordenação do cuidado.

O estudo reforça que, para atender às especificidades dessa população, a equipe CnaR encontra outras formas de apresentar as ofertas de cuidado. Segundo Lemke e Silva ${ }^{30}$, as práticas itinerantes fortalecem a porta de entrada e o acesso das pessoas em situação de rua aos serviços de saúde. A busca ativa é uma variável importante para que a dimensão 'porta de entrada' exista, assim como o acolhimento e ações na rua, como observado. Representa um deslocamento das práticas de cuidado da equipe da unidade de saúde para uma postura ativa e de articulação com o território ${ }^{3}$, que, no caso estudado, é um dispositivo da equipe para enfrentamento das interdições gerenciais.

A dinamicidade do cotidiano das equipes do CnaR supera a lógica das atividades regulares e agendas preestabelecidas. O predomínio de situações imprevisíveis exige dos profissionais criatividade e capacidade resolutiva, que se expressam na 'plasticidade na agenda', uma das principais características do processo de trabalho do CnaR, para além do ponto de vista organizativo, uma vez que a dinamicidade do 'estar na rua' e do inesperado é que modulam o trabalho da equipe 4 .

No campo da intersetorialidade, uma das dimensões da coordenação horizontal21, muitos são os desafios que acabam por limitar a oferta de uma APS mais integral, atenta aos 'modos de andar a vida'13. A rede de relações tecidas pelos profissionais é constantemente desfeita ou enfraquecida pela rotatividade e pela insegurança laboral. A colaboração intersetorial também enfrenta a difícil conciliação de interesses e prioridades das políticas públicas e, sobretudo, das concepções de cuidado e direito da população em situação de rua, explicitado, por exemplo, na tensão em relação ao Resgate Solidário.

A equipe do CnaR vivencia um processo de isolamento na rede, o que também é evidenciado em relação à APS em múltiplos contextos ${ }^{21}$. 
Contudo, não apenas o CnaR é colocado em posição ainda mais marginal em relação ao restante da RAS, mas, sobretudo, seus usuários. A despeito da complexidade e da gravidade das situações de saúde, o sistema de regulação, fluxos e modos de operar da retaguarda terapêutica era o mesmo desenhado para a população domiciliada. Para garantir algum acesso aos serviços de referência, a equipe CnaR se utiliza de projetos terapêuticos e do vínculo com os usuários, inclusive com o acompanhamento destes às consultas especializadas (também com a missão de advocacy) e provisão de meios de transporte.

A negativa de acesso aos demais níveis assistenciais pela exigência de documentos é uma das formas de mitigar o direito à saúde, ainda mais grave no caso da população em situação de rua, cuja retirada representa mais uma face do apagamento de qualquer vestígio de cidadania. A recusa do atendimento nos serviços de saúde, sob a alegação da falta de referência do território, documentos de identificação ou de um responsável pelo cuidado, também é identificada em outros estudos ${ }^{31}$.

\section{Considerações finais}

Argumenta-se que o CnaR pode representar um analisador das redes em geral ao instigar a análise, a explicitação dos elementos de dada realidade institucional ${ }^{32}$, revelando dispositivos potentes e obstáculos à coordenação do cuidado. Ao exigir novos modos de operar os processos de regulação e produção de redes menos burocráticas, na perspectiva da construção de 'redes vivas'33, instiga profissionais e serviços de saúde a produzirem outros agenciamentos nas RAS, para além das redes formais, instruídas por fluxos e regulação que também não atendem às demandas de toda a população. Por outro lado, a coordenação no CnaR explicita que as trajetórias assistenciais não acontecem espontaneamente e são ainda mais necessárias ao cuidado de sujeitos com altos graus de demanda clínica e social.

Produzir redes todo o tempo também constitui tarefa exaustiva para as equipes de saúde. O nomadismo que caracteriza a vida das pessoas vivendo em situação de rua, que não obedece aos códigos sociais vigentes e desafia concepções mais duras que orientam a organização das RAS, instiga a interrogar as concepções mais tradicionais de coordenação do cuidado. Quais arranjos, mais ou menos formais, facilitam ou dificultam a coordenação do cuidado? De toda forma, analisar a coordenação do cuidado a partir da CnaR reafirma seu papel estratégico na tessitura e formação de redes, nas negociações, tensões e desconfortos que pode provocar na RAS ao reconduzir excluídos, ainda que de forma parcial e insuficiente, ao campo da cidadania.

\section{Agradecimentos}

Almeida PF é bolsista de produtividade do Conselho Nacional de Desenvolvimento Científico e Tecnológico (CNPq) e agradece o apoio recebido para o desenvolvimento de estudos sobre o tema.

\section{Colaboradoras}

Santos AR (0000-0002-4857-7410)* e Almeida PF (0000-0003-1676-3574)* contribuíram igualmente para a elaboração do manuscrito. 


\section{Referências}

1. Malta DC, Santos MAS, Stopa SR, et al. A Cobertura da Estratégia de Saúde da Família (ESF) no Brasil, segundo a Pesquisa Nacional de Saúde, 2013. Ciênc. Saúde Colet. 2016; 21(2): 327-338.

2. Brasil. Ministério da Saúde. Política Nacional de Atenção Básica. Brasília, DF: MS; 2012.

3. Londero MFP, Ceccim RB, Bilibio LFS. Consultório de/na rua: desafio para um cuidado em verso na saúde. Interface (Botucatu). 2014; 18(49):251-260.

4. Oliveira RG. Práticas de saúde em contextos de vulnerabilização e negligência de doenças, sujeitos e territórios: potencialidades e contradições na atenção à saúde de pessoas em situação de rua. Saude soc. 2018; 27(1):37-50.

5. Fazel S, Geddes JR, Kushel M. The health of homeless people in high-income countries: descriptive epidemiology, health consequences, and clinical and policy recommendations. Lancet. 2014; 384(9953):1529-40.

6. Fajardo-Bullón F, Esnaola I, Anderson I, et al. Homelessness and self-rated health: evidence from a national survey of homeless people in Spain. BMC Public Health. 2019; 19(1081).

7. Borysow IC, Conill EM, Furtado JP. Atenção à saúde de pessoas em situação de rua: estudo comparado de unidades móveis em Portugal, Estados Unidos e Brasil. Ciênc. Saúde Colet. 2017; 22(3):879-890.

8. Varanda W, Adorno CF. Descartáveis urbanos: discutindo a complexidade da população de rua e o desafio para políticas de saúde. Saude soc. 2004; 13(1):56-69.

9. Brasil. Ministério da Saúde. Portaria no 2.436 , de 21 de setembro de 2017. Aprova a Política Nacional de Atenção Básica, estabelecendo a revisão de diretrizes para a organização da Atenção Básica, no âmbito do Sistema Único de Saúde (SUS). Diário Oficial da União. 22 Set 2017.
10. Prates ML, Machado JC, Silva LS. Desempenho da Atenção Primária à Saúde segundo o instrumento PCATool: uma revisão sistemática. Ciênc. Saúde Colet. 2017; 22(6)1881-1893.

11. Lima JG, Giovanella L, Fausto MCR. Atributos essenciais da Atenção Primária à Saúde: resultados nacionais do PMAQ-AB. Saúde debate. 2018; 42 (esp1):5266.

12. Albuquerque SC. Cuidado em saúde frente às vulnerabilidades: práticas do consultório na rua. 2014. [dissertação]. São Paulo: Pontifícia Universidade Católica; 2014.

13. Silva CGP. Eixos estruturais da práxis em saúde pública do Consultório na Rua POP RUA do Rio de Janeiro. In: Teixeira M, Fonseca Z, organizadores. Saberes e práticas na atenção primária à saúde: Cuidado à população em situação de rua e usuários álcool, crack e outras drogas. São Paulo: Hucitec; 2015. p. 57-68.

14. Teixeira M, Sampaio C, Soares M, et al. Promoção da Saúde: Relato de experiência da equipe de Consultório na Rua do Teias-Escola Manguinhos-ENSP/Fiocruz. In: Teixeira M, Fonseca Z, organizadoras. Saberes e práticas na atenção primária à saúde: Cuidado à população em situação de rua e usuários álcool, crack e outras drogas. São Paulo: Hucitec; 2015. p. 69-86.

15. Trino A, Machado MP, Rodrigues RB. Conceitos norteadores do cuidado junto à população em situação de rua. In: Teixeira M, Fonseca Z, organizadoras. Saberes e práticas na atenção primária à saúde: Cuidado à população em situação de rua e usuários álcool, crack e outras drogas. São Paulo: Hucitec; 2015. p. 2744 .

16. Felinto GM. O Consultório na Rua e a produção de cuidado à população em situação de rua na cidade do Rio de Janeiro. [dissertação]. Rio de Janeiro: Escola Nacional de Saúde Pública Sergio Arouca, Fundação Oswaldo Cruz; 2017. 
17. Wijk LB, Mângia EF. Atenção psicossocial e o cuidado em saúde à população em situação de rua: uma revisão integrativa. Ciênc. Saúde Colet. 2019; 24(9)33573368.

18. Engstrom EM, Teixeira MB. Equipe “Consultório na Rua” de Manguinhos, Rio de Janeiro, Brasil: práticas de cuidado e promoção da saúde em um território vulnerável. Ciênc. Saúde Colet. 2016; 21(6):18391848.

19. McDonald K, Schultz E, Albin L. Care Coordination Atlas Version 4 (AHRQ Publication No. 14-0037-EF). Rockville: Agency for Healthcare Research and Quality; 2014.

20. Vázquez ML, Vargas I, Unger JP, et al. Evaluating the effectiveness of care integration strategies in different healthcare systems in Latin America: the EQUITY-LA II quasi-experimental study protocol. BMJ Open. 2015; 5(7):e007037.

21. Almeida PF, Medina MG, Fausto MCR, et al. Coordenação do cuidado e Atenção Primária à Saúde no Sistema Único de Saúde. Saúde debate. 2018; 42(esp1):244-260.

22. Almeida PF, Fausto MCR, Giovanella L. Fortalecimento da atenção primária à saúde: estratégia para potencializar a coordenação dos cuidados. Revista Panamericana de Salud Pública. 2011; 29(2)84-95.

23. Cadastro Nacional de Estabelecimentos de Saúde. Consulta Estabelecimento-Identificação. [acesso em 2019 set 20]. Disponível em: http://cnes.datasus.gov. br/pages/estabelecimentos/consulta.jsp.

24. Haraway D. Saberes localizados: a questão da ciência para o feminismo e o privilégio da perspectiva parcial. Cadernos Pagu. 1995; (5):7-41.

25. Fernandes JL. O sítio das drogas: etnografia das drogas numa periferia urbana. Lisboa: Editorial Notícias; 1998.

26. Almeida PF, Giovanella L, Mendonça MHM, et al. Desafios à coordenação dos cuidados em saúde: es- tratégias de integração entre níveis assistenciais em grandes centros urbanos. Cad. Saúde Pública. 2010; 26(2):286-298.

27. Casanova AO, Teixeira MB, Montenegro E. O apoio institucional como pilar na cogestão da atenção primária à saúde: a experiência do Programa TEIAS Escola Manguinhos no Rio de Janeiro, Brasil. Ciênc. Saúde Colet. 2014; 19(11):4417-4426.

28. Ornelas AL, Teixeira MGC. Intersetorialidade ou diálogos setoriais? Reflexões a partir da experiência do Projeto Teias-Escola Manguinhos. Rio de Janeiro. Saúde debate. 2015; 39(106):659-670.

29. Bodstein R, Engstrom E, Cardoso G, et al. Produzindo saúde nas ruas: o desafio do trabalho das equipes de Consultório na Rua - Pesquisa de avaliação das práticas das equipes de Consultório na Rua da cidade do Rio de Janeiro. Rio de Janeiro: Fiocruz/ENSP; 2017.

30. Lemke RA, Silva RAN. A busca ativa como princípio político das práticas de cuidado no território. Est. Pesq. Psic. 2010; 10(1):281-295.

31. Borysow IC, Furtado JP. Acesso e intersetorialidade: o acompanhamento de pessoas em situação de rua com transtorno mental grave. Physis. 2013; 23(1):3350 .

32. Rossi A, Passos E. Análise institucional: revisão conceitual e nuances da pesquisa-intervenção no Brasil. Revista EPOS. 2014; 5(1):156-181.

33. Merhy EE, Gomes MPS, Silva E, et al. Redes Vivas: multiplicidades girando as existências, sinais da rua. Implicações para a produção do cuidado e a produção do conhecimento em saúde. Divulg. saúde debate. 2014; (52):153-164.

Recebido em 02/07/2020

Aprovado em 08/10/2020

Conflito de interesses: inexistente

Suporte financeiro: Coordenação de Aperfeiçoamento de Pessoal

de Nível Superior (Capes). Código de financiamento 001 